\title{
Comparative Analysis of DVB System for Different Encoding Techniques with Compressed Images
}

\author{
Amanjot Singh, Himani Sharma* and Manpreet Kaur \\ School of Electronics and Electrical Engineering, Lovely Professional University, Phagwara, \\ Punjab-144411, India; er.ajotsingh@gmail.com, himanis94@gmail.com, mk379544@gmail.com
}

\begin{abstract}
Objectives: In this paper encoding techniques used in the Digital Video Broadcasting-Terrestrial (DVB-T) system have been discussed. The paper focuses upon the comparison between the different types of encodings as a part of the DVB-T standard. Methods/Analysis: Different types of encoding methods which could be the part of the DVB-T standard have been considered.The system used is able to measure and analyze the performance by using different encoding techniques along with the Orthogonal Frequency-Division Multiplexing (OFDM). Findings: DVB-T was developed by the Digital Video Broadcasting (DVB) Project that was basically for broadcasting. The compressed images, digital audio, compressed videos and other forms of data in Moving Picture Expert Group (MPEG) transport stream, through OFDM modulation is transmitted by this DVB-T system. Novelty /Improvement: In the DVB-T Reed Solomon encoding is used to encrypt the data. Hybrid coding has been proposed as the way to improve the performance.
\end{abstract}

Keywords: Convolution Encoding, Digital Video Broadcasting- Terrestrial (DVB-T), OFDM, Rs Coding

\section{Introduction}

Wireless communication is a one of the communication system that delivers multimedia and other data services to the users having smart devices capable of maintain wireless link ${ }^{1}$. Today's wireless based communication system Standards are capable of broadcasting and delivering services related videos and images almost globally. Users equipped with any kind of wireless communication devices increasingly need to connect to the network and required to receive multimedia data, messages, data queries and transfer file. One of the standards used in delivering multimedia data is DVB-T system. In this work DVB-T system has been considered under study. DVB-T is based on the multicarrier modulation technique called as OFDM. OFDM stands for orthogonal frequency division multiplexing 2 . It is a multicarrier modulation technique that has newly found its wide implementation in a wide variety of high data rate communication system, including Digital Subscriber Lines (DSL), wireless Local Area Network (LAN), digital video broadcasting, now
Wi-max and many other emerging wireless broadband systems. OFDM's popularity for high data rate application is mainly for its efficient and elastic management of Inter Symbol Interference (ISI) under very dispersive channels ${ }^{3,4}$. The OFDM system has the advantage of the resistance against fading effects and has high spectrum efficiency ${ }^{5,6}$. OFDM has its applications in wireless applications such as DVB-T system. Along with basic OFDM there are various other coding techniques which are the part of the DVB-T Standard. These techniques are useful in handling the issues related to OFDM. Moreover, it has also increased the quality of the DVB system. In the paper, Section 1 is related with Introduction, Section 2 is giving the details of the DVB system, Section 3 is dealing with Analysis and Simulation, Section 4 is giving the conclusion.

\section{DVB-T System}

Wireless communication is undergoing in the tremendous growth and broad implementations in a wide variety

${ }^{*}$ Author for correspondence 


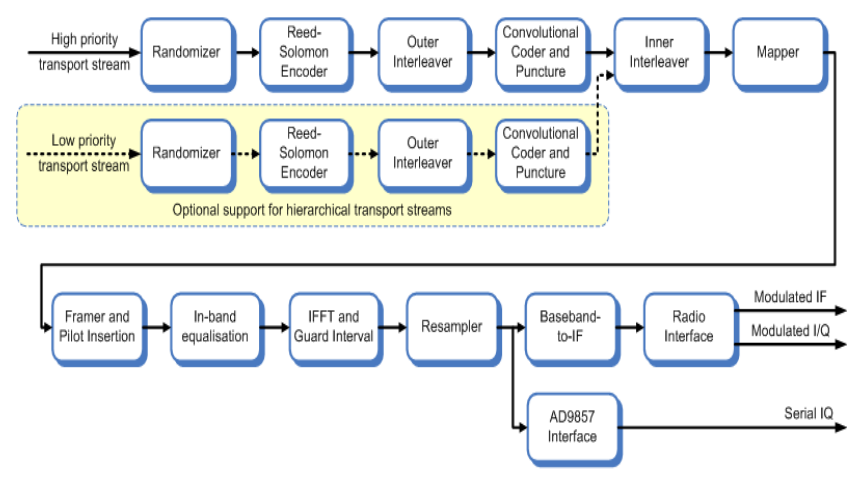

Figure 1. DVB-T block diagram 21 .

of the applications. As there is an increasing in the worldwide interest in low profile, low-cost, light weight and wideband system designs, nowadays OFDM has become an attractive system for transmission and one of the applications of OFDM is DVB-T. DVB-T, it is the designation specified for the terrestrial transmission system. It was firstly used in broad casting in the UK in 1997 and was settled by DVB project. DVB-T is acceptable standard in various countries ${ }^{7}$. The DVB structure was introduced in 1993 by the European Telecommunications Standards Institute (ETSI) with the objective of standardizing propelled TV organizations. The principal step was that the structure would contain a blend of picture, sound or media. This work was achieved with ETSI models for the physical layers ${ }^{8}$. The framing structure, modulation for Digital Terrestrial Television (DTT) broadcasting and channel coding, all these specifications are the part of DVB-T. It is using the very famous OFDM multicarrier modulation technique. However, the DVB-T standard has very flexible system for technical characteristics. In the paper, work is focused on coding techniques along with OFDM. Performance with a single encoding and with the hybrid encoding has been considered. Reed Solomon and convolution encodings are used with OFDM as shown in Figure 1. DVB-T also nominates three modulation techniques that are QPSK, 16QAM, 64QAM? .

The one hybrid encoding pair is between RS encoding and convolution encoding and the other hybrid encoding can be done by combining hamming and convolution using OFDM.

\subsection{Reed Solomon Encoding}

The RS encoder encodes the message in the input vector using an $(\mathrm{N}, \mathrm{K})$ Reed Solomon encoder with narrow sense generator polynomial. The input must be a frame based col- umn vector with an integer multiple of k elements. The high power amplifier is a complex baseband model of amplifier with noise. The amount of noise added to the output signal may be specified in terms of noise temperature, noise figure or noise factor. The white Gaussian noise is added to the input signal. The received input signal is decoded by RS decoder. Integer to bit converter, map a vector of integer valued. The outer error protection is implemented with a byte-oriented block code. For each block, i.e., the TS packet - error correcting bits are calculated. The result is a block of correction bytes that is appended to the TS packet. The block code that is used in Reed-Solomon is (255 239) code. That means that 16 correction bytes are appended to the 239 information bytes ${ }^{10}$. The outer Interleaver that follows does not provide any additional error correction capability, but rearranges bytes in order to facilitate the correction of long burst errors. A block Interleaver that reads the bytes into a storage matrix line by line and is read out column by column would be one possible implementation. Such a block Interleaver shows disadvantages regarding storage capacity required, synchronization, and sensitivity against periodic disturbances.

RS codes are basically defines the data in K data symbols which calculates the N-K symbols, add on the K data symbol for $\mathrm{n}$ symbols to parity symbols. Reed Solomon codes are considered to be the important case for higher stages of $\mathrm{BCH}$ codes ${ }^{10}$. The encoder is basically shift register having $\mathrm{m}$ bits width of every single register. The coefficient of the RS generator polynomial is the coefficient of multiplier coefficient. According to architectural, the encoder signifies the set of shift registers. From the gallows arithmetic, the set of shift registers is attached by multiplier and integrators. The shift register characterizes the bits and every bit covers any one element of the Galois Field (GF). Symbols are placed in a particular location and are transmitted to the output line $\mathrm{e}^{11}$. Simultaneously, the symbol is loaded into position from the input line as strictly defined time intervals ${ }^{12}$. A Reed Solomon decoder is placed at the receiving side which decodes the code. It basically works to find out the location of the error and further remove or correct the error. Reed Solomon coder considered to be the perfect for correcting the burst error.

\subsection{Convolution Codes}

There are various types of channel coding are being used, but convolution codes are considered to be a better one. Convolution codes contain three basic parameters that 
are $\mathrm{n}, \mathrm{k}, \mathrm{K}$; where, $\mathrm{K}$ indicates the constraint length that depends upon the total number of shift registers, $\mathrm{n}$ indicates the number of output bits, $\mathrm{k}$ is the number of input bits. In convolution codes, the data is coming in the form of a continuous stream of data. In these codes, the encoder has an arrangement structure which is similar to block codes ${ }^{15}$. Convolution code mapping is bijective and it is a linear code. At the receiving side Viterbi decoder is used which decodes the incoming data. As block codes are good against burst error, so convolution codes can be used along with block codes, to increase the efficiency. In speech or image like transmission convolution codes are may be employed where low-latency is needed.

\subsubsection{Convolution Interleaver}

Interleaving is frequently used in digital communication and storage systems to improve the performance of forward error correcting codes $\frac{13}{}$. If the number of errors within a code word exceeds the error-correcting code's capability, it fails to recover the original code word ${ }^{13}$. Interleaving ameliorates this problem by shuffling source symbols across several code words, thereby creating a more uniform distribution of errors. Therefore, interleaving is widely used for burst error-correction. Error free data transmission can be attained by the proper choice of code error for error detection ${ }^{14}$. The feedback channel has to be practically noiseless. This is not a severe restriction. The information transfer over the feedback channel is at a low rate, a substantial amount of redundancy can be used to ensure reliable feedback transmission. A straightforward and effective method to apply coding on the burst error channel is to use interleaving. With this method, the channel is effectively transformed into an independenterror channel for which many forward-error correction coding techniques are applicable. In the work the data is encoded using a convolutional encoder with a constrained length 8 bit data as input and a convoluted output of 16 bits. The design rate of convolutional encoder is $\mathrm{Rc}=$ $1 / 2(\mathrm{Rc}=\mathrm{k} / \mathrm{n})$, where $\mathrm{k}=1$ (number of inputs) and $\mathrm{n}=2$ (number of outputs). The transmitter includes an encoder followed by an Interleaver that scrambles the encoded data stream in a deterministic manner. The interleaved data are transmitted through the bursty channel. In the receiver, a de-interleaver is used to perform the inverse operation, thus the received data are unscrambled, so that the decoding operation may proceed properly. The combination of interleaving and forward- error correction thus provides an effective means of combating the effect of error bursts.

\subsection{Other Encoding Techniques}

Low Density Parity Codes (LDPC) are a linear correcting method that is used for transmitting the data over noisy transmission channel. LDPC coding can be implemented by using a sparse bipartite graph. LDPC codes are used to transmit highly efficient information over bandwidth in the presence of noise. LDPC can be used for the purpose of error correcting in the DVB for the satellite transmis$\operatorname{sion}^{16}$. The Sparse bipartite graph structure is a one type of the property that allows better efficiency of LDPC code. In the wireless communication, hamming codes are a family of linear error correcting codes that generalize the hamming $(7,4) \operatorname{code}^{1 \frac{17}{}}$. It was invented by Richard hamming in 1950. Basically, hamming codes encode four data bits into 7 bits by adding three parity bits. The major applications of hamming codes are DRAM memory chips and satellite communication hardware.

\subsection{Hybrid Encoding}

Hybrid encoding can be defined as the combination of two or more encoding techniques. There are various encoding techniques which are being used. Hybrid encoding double encrypt the data and secures the information while transmission ${ }^{18}$. Here convolution encoding as well as Reed Solomon encoding has been used. Hybrid encoding shows the less BER as compare to convolution encoding. The first hybrid encoding pair that can be used is between RS encoding and convolution encoding and the second hybrid encoding can be done by combining hamming and Convolution along with OFDM. Firstly the RS encoder are used, the output of RS encoder has been passed to the convolution encoder as an input. Hybrid encoding is used to enhance the performance of the system.

As per today's requirements the data rates for transmissions are high. Therefore, double encoding is one of the best ways of maintaining good quality links. In the base approach only one encoder was in use that was an RS encoder; however in the proposed scheme two or more encoding schemes may be used i.e. RS encoding along with other encoding. There is PRBS encoding that is pseudo random bit sequence can also be combined with the RS encoder and other detecting techniques. It also adds redundant bits to the original data so that the data remain secure from outside users. This proposed method 
ensures the robustness and fidelity of the improved DVB-T system for broadcasting the data with a fixed or moving receiver.

\section{Analysis and Simulation}

Reed Solomon coding is a subset of BCH codes and linear block codes. It is used to improve the bit error rate. It is used in the applications of high speed communication. Other applications are Compact Disc (CD) controller application, Digital Versatile Disc (DVD) controller applications, optical fiber system and Cellular telephone system. Another technique like LDPC codes are used for fast encoding and decoding algorithms. These codes are generally designed in such a way for algorithms that are generally being used able to recover the original data which is added to parity bits. It makes LDPC not only attractive from a theoretical point of view, but also perfect for practical applications like DVB. Already LDPC codes were used for deep space applications.

Convolutional codes are used for error detection. One of the major applications of convolutional encoding is satellite communication. In the satellite communication power and bandwidth are two major constraints, so there is often need of better error control coding to improve better quality of transmission, so convolution encoding is a better option for the application including satellite links. Therefore, already in DVB based systems it is being used. In satellite communication convolutional codes with constraint length $\mathrm{k}=7$ is widely used ${ }^{19}$. This coding technique does have other applications such as mobile communication and broadcasting purpose applications because this coding technique is efficient for the reliable wireless transmission.

Hamming codes are oldest error correction codes, because of its fair distance property, good algebraic structure; it is still used in the field of communications. There are numerous applications of hamming codes such that public safety mobile communications system, digital mobile radio ${ }^{20}$. Hamming codes are used in the trellis diagram to find out the minimum distance for the decoding the convolutional codeword. Hamming codes also used in the neural network field to find out the minimum acceptable distance between the incoming and outgoing nodes. Therefore, it can be included in the DVB-T system to improve the quality of communication.

Hybrid encoding technique is a combination of encoding methods can be used in the field of multimedia based applications. Hybrid encoding may also include the other applications such that to encode the video, background subtraction, phase tracking and compression of data as a part of DVB extensions.

\subsection{Simulation}

In order to analyse the performance of DVB-T system, MATLAB based simulated system has been considered. An original compressed image as data is transmitted through the DVB based system and following results have been obtained with different parameters.

The reference compressed image (Figure 2.) is taken as an input to analyse the performance of the system using different encoding techniques. In the simulation both single and hybrid encoding has been considered. It has been seen that hybrid coding based on double encoding provides better picture quality at receiver.

\subsubsection{DVB-T for single encoding}

The above result is demonstrated output for DVB-T system. The data to be taken is a compressed image. Two dimensional data was sent with encrypted coding under

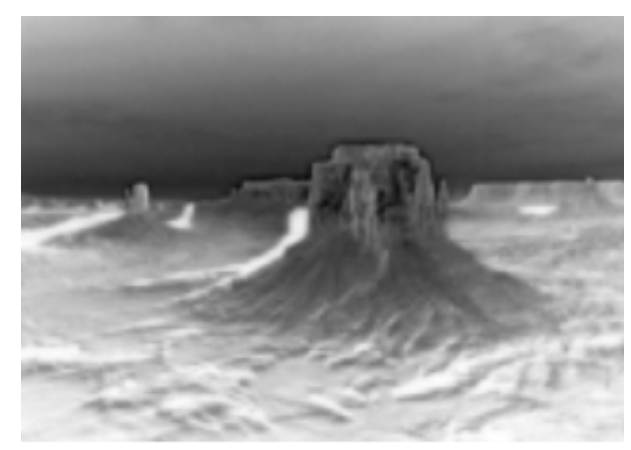

Figure 2. Reference compressed image.

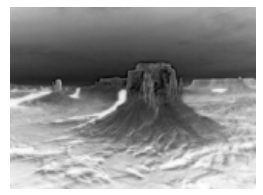

Figure 3. Output of DVB-T for single encoding with code rate $1 / 2$, error ratio 91.6667 .

Table 1. Output parameters for DVB-T system

\begin{tabular}{|c|c|c|c|c|}
\hline Parameters & Error Ratio & Mean & PSNR & Time \\
\hline Values & 91.6667 & 14.7686 & 36.43739 & 22.28341 \\
\hline
\end{tabular}




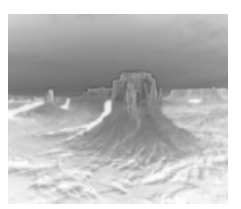

Figure 4. Output of DVB-T for double encoding with a code rate $1 / 2$, error ratio 47.2379 .

Table 2. Output parameters for improved DVB-T system

\begin{tabular}{|l|l|l|l|l|}
\hline Parameters & Error ratio & Mean & PSNR & Time \\
\hline Values & 47.2379 & 17.5538 & 35.6870 & 35.17969 \\
\hline
\end{tabular}

effective Burst error as simulated by user replicating the actual situation of system in run time. Here, the error ratio is $91.6667 \%$ which means the signal quality is not good as the bit error ratio and the signal to noise ratio are inversely proportional to each other. More is the error ratio, lesser is the signal quality. The code rate is taken $1 / 2$ because it has got highest redundancy and transportation immunity. This mode can be exercised on robust distributed mediums.

\subsubsection{DVB-T for double/Hybrid encoding}

Figure 4. Demonstrates the output for a DVB-T system with hybrid coding. Again, the data taken was a compressed image of same dimension as in previous. The result shows the reduction of bit error ratio. Here, the result shows that the bit error ratio is reduced, i.e. $47.2379 \%$ than the previous approach. Based on these results further conclusion can be given as further.

\section{Conclusion}

As per the study it has been seen that a good encoding technique along with OFDM can provide an acceptable data transfer rate in a wireless environment. DVB-T is a working standard, which is including these as technical specifications. The DVB-T standard is a long range content transmission and reception system under a highly encoded and encrypted environment. However, still its performance can be further improved with improvements in coding techniques for error corrections as well as for the loss in data with the noise issues arising from fading and non-synchronization of data between user and transmission station. There were certain improvements in DVB-T with Hybrid coding scheme and also to reduce the interference an additional encoding can be included. The simulation shows the reduction in the overall error ratio. In today's time there is DVB-T2 standard available for transmission. In future, there is scope of improvement in system with improved coding techniques which could be combined with MIMO systems for DVB-T applications.

\section{References}

1. Han SH, Lee JH. An overview of peak-to- average power ratio reduction techniques for multicarrier transmission. IEEE Wireless Communications. 2005; 12(2):56-65.

2. Lasorte N, Barnels W, Refai H. The History of OFDM. IEEE Communication; 2008.

3. Zhang L, Thibault L, Baudreau R, Wu Y. A low complexity robust OFDM receiver for fast fading channels. IEEE Transactions on Broadcasting. 2014 Jun; 60(2):347-57.

4. Singh A, Kaur H. Non linearity analysis of high power amplifier in OFDM system. International Journal of Computer Applications. 2012 Jan; 37(2):37-41.

5. Kumar BS, Kumar KRS, Radhakrishnan R. An efficient inter carrier interference schemes for OFDM systems. International Journal of Computer Science and Information Security (IJCSIS). 2009; 6(3):141-8.

6. Prasad, Siddaiah P, Reddy L, Lekha K. Analysis of fast fading in wireless communication channels. International Journal of Science and Technology (IJST). 2010; 3(1):139-45.

7. DVB-T single frequency network architecture. Spectracom Corporation; 2007.

8. Hanzo L, Cherriman P, Streit J. Video compression and communications: from basics to H.261, H.263, H.264, MPEG2, MPEG4 for DVB and HSDPA-Style adaptive turbo-transceivers. IEEE Press; 2007.

9. Deksnys V, Citavicim A. Simulation of lithuanian version of DVB-T system. EE. 2009; 8(96).

10. Shrivastava P, Singh U. Error detection and correction using reed solomon codes. International Journal of Advanced Research in Computer Science and Software Engineering (IJARCSSE). 2013 Aug; 3(8):965-9.

11. Guruswami V, Sudan M. Improved decoding of ReedSolomon codes and algebraic geometry codes. IEEE Transactions on Information Theory, 1999 Sep; 45 (6): 1757-67 DOI:10.1109/18.782097

12. Kaur M. Study of reed solomon encoder. International Journal of Innovative Research in Computer and Communication Engineering (IJIRCCE). 2013 Apr; 1(2): 190-2.

13. Taub H, Schilling DL, Saha G. Taub's principles of communication systems. edition-3, Chapter 13, Information theory and coding. 2014 Nov 15; 3(13):682-4. 
14. Haykin S. Digital modulation techniques. Chapter 8, Error-Control Coding. Communication Systems, Wiley Publication, 3rd Edition 2004.

15. Arshad N, Basit A. Implementation and analysis of convolutional codes using MATLAB. International Journal of Multidisciplinary Sciences and Engineering. 2012 Aug; 3(8):9-12.

16. Fiedler J. Hamming Codes [Internet]. 2004 [cited 2004]. Available from: www.orion.math.iastate.edu/linglong/ Math690F04/Hamming codes.pdf.
17. Keiser BE, Strange E. Digital telephony and network integration; 1995.

18. Bhargava V.K, Q. Yang, D.J. Peterson. Coding theory and its application in communication systems. Defense Science Journal. 1993 Jan; 43: 59-69.

19. Lim J, Lee J, Shin M, Song YJ. Applications of Hamming codes to Public Safety Mobile communication system. Advanced Science and Technology Letters. 2014; 51:52-5.

20. Block diagram of the DVB-T modulator .www.commsonic. com/products/TDvbTModulatorDiagram.htm 